\title{
Cortilivo o cortilizio? Vanno bene entrambi!
}

\section{Vittorio Coletti}

PUBBLICATO: 03 MARZO 2020

\section{Quesito:}

Sono arrivati vari quesiti sugli aggettivi cortilivo e cortilizio, usati spesso nel linguaggio tecnico dell'edilizia per riferirsi al cortile o a una parte comune di un condominio. Sono entrambi corretti? E presentano differenze di significato?

\section{Cortilivo o cortilizio? Vanno bene entrambi!}

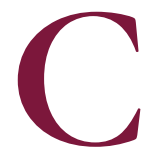

ominciamo col dire che cortilivo e cortilizio hanno lo stesso significato ('del cortile, destinato a cortile', quindi di proprietà comune), uguale legittimità etimologica (-ivo e -izio sono suffissi che possono formare aggettivi da sostantivi col valore di 'pertinente a, destinato a', come, rispettivamente, in arbustivo e cardinalizio), ma stranamente diversa fortuna lessicografica.

Cortilivo è attestato nel supplemento del GDLI 2004, registrato dal GRADIT e dallo Zingarelli con data 1983 e classificazione di raro e burocratico. In realtà, grazie a Google libri, ne troviamo attestazione già in un testo del 1767 , L'agrimensore instruito di F.M. Girri, dove si parla di "fabbriche rustiche, unite al terreno casamentivo, cortilivo etc." che "fruttano il cinque per cento" (da notare, nel passo, anche la presenza di casamentivo, altro aggettivo tecnico non registrato nei dizionari, che ha come precedente il latino casamentivum documentato in testi medievali).

Cortilizio invece non è accolto nei dizionari, ma c’è una sua attestazione già nel i695, negli Annali, memorie ed Homini illustri di Sangimignano di G.V. Coppi, dove però è usato come sostantivo, per dire 'terreno di proprietà', se non è un refuso per fortilizio ("divieto di dare ricetto a banditi nel suo cortilizio"); come aggettivo, in ogni caso, compare tra le leggi pubblicate sulla "Gazzetta di Parma" del I848, in cui si parla di un "Podere arativo, alberato, vitato, prativo, caneparivo [sic, ma forse da correggere in canapativo 'in cui si può coltivare la canapa'], cortilizio".

Entrambi gli aggettivi sono spesso associati ad area (cortiliva o cortilizia), anche in leggi e sentenze recenti, che definiscono in genere caratteristiche e destinazione di spazi comuni a caseggiati. In A. Cuoghi, La vigilanza edilizia, 2015 area cortiliva riceve una precisa definizione: "si definisce area cortiliva di una o più unità edilizie la superficie scoperta, catastalmente o progettualmente individuata, posta al diretto servizio delle unità edilizie stesse". Il sito Zanichelli DizionariPiù segnala un'ordinanza del 1990 del Comune di Lugo che fa "divieto di immettere gatti nell'area cortiliva del padiglione ospedaliero".

Non ho trovato una definizione altrettanto precisa di area cortilizia, ma questo non significa che l'aggettivo, come si è visto, non sia frequente anche in testi giuridici e loro commenti, specie in altre collocazioni usuali, come terreno e spazio (cortilivo o cortilizio). Di terreno cortilizio parla una sentenza della Cassazione del 2orr.

Cortilizio per altro è molto più frequente di cortilivo su Google, e imperversa negli annunci immobiliari ("Villetta con ampio spazio cortilizio").

Negli impieghi formali, burocratici e giuridici, cortilivo e cortilizio sono riferiti soprattutto alla proprietà e come tali sono pressoché sinonimi di condominiale; in questi contesti non sarebbe male 
sostituirli appunto con questo diffuso aggettivo o addirittura con comune. Quando, invece, come negli annunci di compravendita di immobili, sono riferiti anche (o solo) alla collocazione del terreno, dell'area, mantengono un più forte legame con l'etimo di cortile (spazio interno aperto tra edifici) da cui discendono e sono meno sostituibili. In questo caso prevale per quantità cortilizio. È dunque singolare e ingiusta la diversa fortuna lessicografica dei due aggettivi. Anche il correttore automatico segnala come erroneo cortilizio e andrebbe quindi aggiornato.

In conclusione, cortilivo e cortilizio sono entrambi legittimi e meritano lo stesso trattamento, anche se si spera che i sintagmi in cui più spesso si trovano finiscano per trovare una definizione precisa che attribuisca l'uno o l'altro aggettivo ai diversi sostantivi con cui si incontrano, specie nelle cause civili e nei regolamenti. Ma per il momento sono, ripeto, legittimi e sinonimi, anche se a cortilizio spetta un risarcimento lessicografico che è giusto auspicare dall'Accademia che ha inaugurato la tradizione dei grandi vocabolari di italiano.

\section{Cita come:}

Vittorio Coletti, Cortilivo o cortilizio? Vanno bene entrambi!, "Italiano digitale", 2019, XII, 2020/1 (gennaio-marzo)

DOI: $10.35948 / 2532-9006 / 2020.3247$

Copyright 2019 Accademia della Crusca

Pubblicato con licenza creative commons CC BY-NC-ND 